\title{
Genomic analysis of bifunctional Class C-Class D $\beta$-lactamases in environmental bacteria
}

\author{
Melise Chaves Silveira ${ }^{1 /+}$, Marcos Catanho², Antônio Basílio de Miranda1 \\ ${ }^{1}$ Fundação Oswaldo Cruz-Fiocruz, Instituto Oswaldo Cruz, Laboratório de Biologia Computacional e Sistemas, Rio de Janeiro, RJ, Brasil \\ ${ }^{2}$ Fundação Oswaldo Cruz-Fiocruz, Instituto Oswaldo Cruz, Laboratório de Genômica Funcional e Bioinformática, Rio de Janeiro, RJ, Brasil
}

$\beta$-lactamases, which are found in several bacterial species and environments, are the main cause of resistance to $\beta$-lactams in Gram-negative bacteria. In 2009, a protein (LRA-13) with two $\beta$-lactamase domains (one class $\mathrm{C}$ domain and one class D domain) was experimentally characterised, and an extended action spectrum against $\beta$-lactams consistent with two functional domains was found. Here, we present the results of searches in the non-redundant NCBI protein database that revealed the existence of a group of homologous bifunctional $\beta$-lactamases in the genomes of environmental bacteria. These findings suggest that bifunctional $\beta$-lactamases are widespread in nature; these findings also raise concern that bifunctional $\beta$-lactamases may be transferred to bacteria of clinical importance through lateral gene transfer mechanisms.

Key words: bifunctional $\beta$-lactamase - antibiotic resistance - health surveillance

$\beta$-lactamases are part of a large group of diverse and widely distributed enzymes, encoded by genes located on both the chromosome and on mobile genetic elements (Bush 2001, Srivastava et al. 2014). Bacteria containing $\beta$-lactamases have been found in a wide range of environmental conditions, including soil, water and in human and animal microbiota (Allen et al. 2009, Gibson et al. 2015, Fróes et al. 2016). The production of bacterial $\beta$-lactamases is the main cause of $\beta$-lactam resistance in Gram-negative bacteria (Bush 2001), and it is essential to know their spectrum of action and distribution (Bush 2001, Gibson et al. 2015).

A few years ago, a novel $\beta$-lactamase, LRA-13, was identified in the metagenome of uncultured bacteria isolated from Alaskan soil (Allen et al. 2009). LRA-13 contains two serine- $\beta$-lactamase domains - one belonging to class $\mathrm{C}$ and one to class $\mathrm{D}$. The fusion of these domains expands the hydrolytic capacity of the protein beyond what either could display alone, thereby causing resistance to amoxicillin, ampicillin, cephalexin (class C) and carbenicillin (class D), as demonstrated experimentally (Allen et al. 2009). The identification of bifunctional $\beta$-lactamases in other bacterial species may indicate that more attention should be given to genes encoding this class of enzyme, particularly since lateral gene transfer events are common among prokaryotes (Soucy et al. 2015), and these genes could theoretically transfer to human bacterial pathogens.

To determine whether bifunctional $\beta$-lactamases are present in other bacterial species, we searched the nonredundant NCBI protein database (July 2017) utilising

doi: 10.1590/0074-02760180098

MCS was supported by CAPES.

+ Corresponding author: melisechaves@gmail.com

Received 23 February 2018

Accepted 20 April 2018 the BLAST programme (Altschul et al. 1997) to identify potential homologs of the LRA-13 enzyme. We identified nine putative homologs encoded in the genomes of nine different bacterial species or isolates (Table). The sequence of these nine proteins is highly conserved between the nine species $(\geq 94 \%)$ and align closely with the reference sequence of LRA-13 ( $\geq 65 \%$ ). All nine proteins have two complete characteristic domains of class C (COG1680, PRK11289) and one of class D (COG2602) according to the Conserved Domain Database (CDD, Batch CD-search tool) (Marchler-Bauer et al. 2017). In addition, these proteins display characteristic active site patterns of both class C (PS00336) and class D (PS00337) domains according to PROSITE (Sigrist et al. 2012), including the serine (S) catalytic residue.

We then examined whether these bifunctional $\beta$-lactamases are encoded in genomic islands or near prophage sequences using IslandViewer 4 (Bertelli et al. 2017), which integrates four different genomic island prediction methods, and the PHAge Search Tool (PHAST) (Zhou et al. 2011), which identifies prophage sequences in bacterial genomes. Bifunctional $\beta$-lactamase was not encoded in genomic islands, and only the strain Massilia sp. Root351 showed the presence of an incomplete $8.4 \mathrm{~Kb}$ prophage located approximately $3.4 \mathrm{~Kb}$ downstream from the gene encoding a bifunctional $\beta$-lactamase.

The $\beta$-lactamases with fused domains found in this work were identified in the genomes of bacterial strains belonging to three distinct Gram-negative genera (Baldani et al. 2014): Duganella spp., Janthinobacterium sp. and Massilia sp. The original annotation of the gene products encoding these enzymes is either "class $\mathrm{C}$ $\beta$-lactamase" or "class D $\beta$-lactamase" (Table). In all cases, the upstream protein-coding gene is originally annotated as "class D $\beta$-lactamase", and their products display a complete characteristic domain of class D (COG2602). Additionally, these products display complete or incomplete domains of methicillin resistance regulatory proteins BlaR1 and MecR1 (COG4219, 


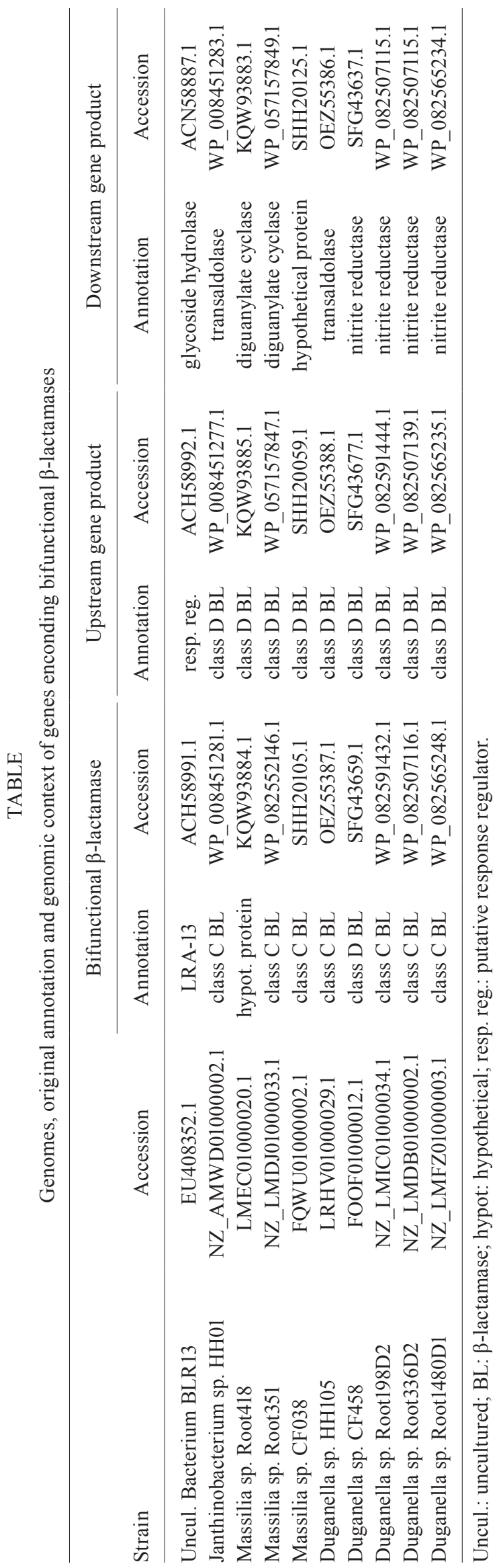

cd07341), corresponding to the structure of the signaltransducing integral membrane protein that regulates the $\beta$-lactam resistance in the Gram-positive species Staphylococcus aureus (Wilke et al. 2004). Genes located downstream of the bifunctional $\beta$-lactamase vary among distinct bacterial genera and fall into four functional categories: "diguanylate cyclase", "transaldolase", "glycoside hydrolase" or "hypothetical" (Table). All Massilia strains harbor a gene encoding a transcriptional regulator which is upstream and inverted relative to the gene encoding the bifunctional $\beta$-lactamase. This transcriptional regulator mediates the expression of the regulatory protein BlaR1, which is also inverted in relation to the bifunctional $\beta$-lactamase (Figure).

The strains Janthinobacterium sp. HH01 and Duganella sp. HH105 were isolated from an aquatic environment and exhibit an ampicillin resistance phenotype (Hornung et al. 2013, Haack et al. 2016). The strains Duganella sp. CF458 (Gp0136797) and Massilia sp. CF038 (Gp0136806) were isolated in 2016 from the root of a Populus tree in Tennessee, USA (NCBI BioProject PRJEB18228), while the other strains of Duganella sp. and Massilia sp. were isolated from the Arabidopsis root microbiota (Bai et al. 2015). These three genera belong to the family Oxalobacteraceae (Betaproteobacteria group); they are (supposedly) non-pathogenic to humans, animals and plants and are known for their antifungal effect (Yin et al. 2013, Haack et al. 2016). Bacteria from this family have few phenotypic differences, and their classification in distinct genera is mainly based on $16 \mathrm{~S}$ rRNA gene sequencing (Kämpfer et al. 2007). Functional metallo- $\beta$-lactamases (class B) have already been described in Janthinobacterium lividum and Massilia oculi (Docquier et al. 2004, Gudeta et al. 2016). These genes are phylogenetically related and share common ancestors with acquired $\beta$-lactamases produced by clinical pathogens, which could have been acquired from members of Oxalobacteraceae (Gudeta et al. 2016).

According to Allen et al. (2009), the LRA-13 $\beta$-lactamase appears to be the result of an ancient natural fusion of genes encoding complete enzymes, not due to modern selective pressure caused by the extensive use of antibiotics. In two cases, the bifunctional $\beta$-lactamase sequences are virtually identical (Duganella sp. Root 198D2 vs. Duganella sp. Root 336D2, and Duganella sp. HH105 vs. Janthinobacterium sp. HH01) with 99\% and $96 \%$ amino acid identity over their entire sequences, respectively. However, LRA-13 is not the only example of a bifunctional enzyme implicated in antibiotic resistance. Some aminoglycoside transferases are capable of conferring resistance to practically all antibiotics of this class via modifications to the antibiotic molecule at two different sites. However, unlike LRA-13, their origin appears to be recent and caused by the clinical (mis)use of aminoglycosides (Kim et al. 2007, Zhang et al. 2009).

The absence of genes encoding bifunctional $\beta$-lactamases in genomic islands or near prophage sequences, and the fact that these genes are shared among all currently sampled representatives of three genera belonging to the same family (Oxalobacteraceae), suggest that, similar to LRA-13, the gene fusion might have oc- 


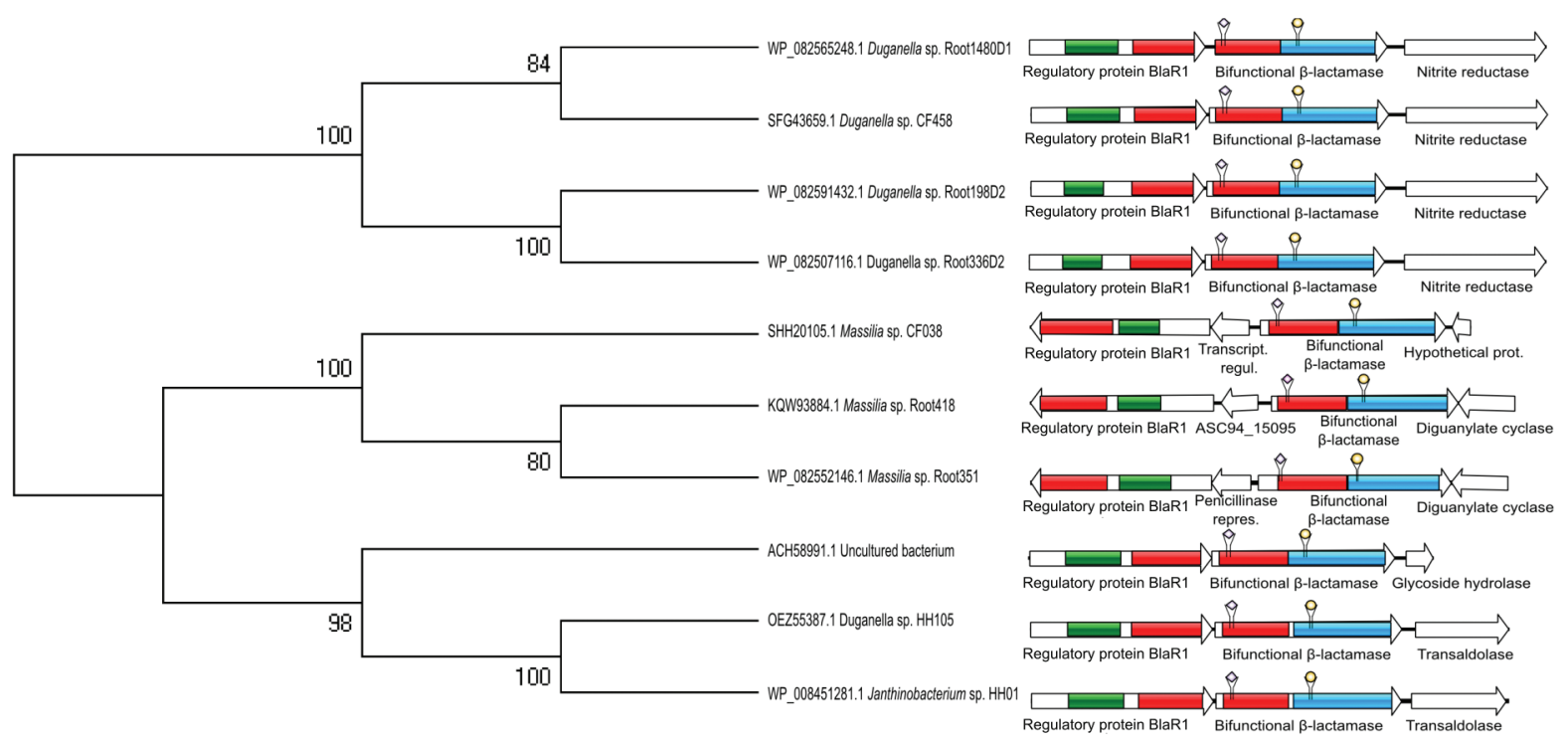

Phyletic pattern of genes encoding bifunctional $\beta$-lactamases, and their genomic organisation including surrounding genes. Left: a dendrogram representing the relationships between the bifunctional $\beta$-lactamases in this study. Right: a panel displaying the order and orientation of the genes encoding the bifunctional $\beta$-lactamases and surrounding genes. The boxes represent distinct domains: red, class D; green, MecR1/BlaR1; blue, class C. Arrows indicate gene orientation. Diamonds and circles above the bifunctional $\beta$-lactamases indicate the location of class D and class $\mathrm{C}$ active sites, respectively. Sequences were globally aligned using MAFFT version 7 (Katoh et al. 2017). The dendrogram was constructed with MEGA version 7 (Kumar et al. 2016), applying the NJ algorithm and 500 bootstrap replicates. The panel containing genes, domains and active sites was drawn using the IBS (Liu et al. 2015).

curred naturally and long ago. Indeed, several benefits of bearing a bifunctional enzyme can be assumed, such as the concomitant mobilisation of two different functions, the potential for complementary and extended resistance, and the simultaneous selection of two enzymatic activities by the selective pressure exerted by a single antibiotic (Zhang et al. 2009).

The evidence presented here suggests that bifunctional $\beta$-lactamases are part of a new class of enzyme with potentially broad spectrums of action. The first reported enzyme within this class (LRA-13) was found in a non-cultivable bacterium from a remote soil sample, but proteins with the same characteristics can be found in different bacterial genera present in water, soil, and even sharing the same niche. To date, there is no evidence of a clinically significant role for bifunctional $\beta$-lactamases, but this possibility cannot be ignored. Chromosomal location and degree of sequence conservation suggest that these enzymes might be characteristic of the family Oxalobacteraceae. Since our knowledge of the environmental microbiota is far from complete, it is necessary to examine the eventual dissemination of these bifunctional $\beta$-lactamases to bacteria that are pathogenic to humans and other animals.

\section{AUTHORS' CONTRIBUTION}

MCS and ABM conceived of the manuscript outline; MCS, MC and ABM wrote, edited and revised the manuscript.

\section{REFERENCES}

Allen HK, Moe LA, Rodbumrer J, Gaarder A, Handelsman J. Functional metagenomics reveals diverse $\beta$-lactamases in a remote Alaskan soil. ISME J. 2009; 3(2): 243-51.
Altschul SF, Madden TL, Schäffer AA, Zhang J, Zhang Z, Miller W, et al. Gapped BLAST and PSI-BLAST: a new generation of protein database search programs. Nucleic Acids Res. 1997; 25(17): 3389-3402.

Bai Y, Müller D, Srinivas G, Garrido-Oter R, Potthoff E, Rott M, et al. Functional overlap of the Arabidopsis leaf and root microbiota. Nature. 2015; 528(7582): 364-9.

Baldani JI, Rouws L, Cruz LM, Olivares FL, Schmid M, Hartmann A. The family Oxalobacteraceae. In: Rosenverg E, DeLong EF, Lory S, Stackebrandt E, Thompson F, eds. The prokaryotes - alphaproteobacteria and betaproteobacteria. Berlin/Heidelberg: Springer-Verlag; 2014. p. 919-74.

Bertelli C, Laird MR, Williams KP, Lau BY, Hoad G, Simon Fraser University Research Computing Group, et al. IslandViewer 4: expanded prediction of genomic islands for larger-scale datasets. Nucleic Acids Res. 2017; 45(W1): W30-5.

Bush K. New $\beta$-lactamases in gram-negative bacteria: diversity and impact on the selection of antimicrobial therapy. Clin Infect Dis. 2001; 32: 1085-9.

Docquier JD, Lopizzo T, Liberatori S, Prenna M, Thaller MC, Frère $\mathrm{JM}$, et al. Biochemical characterization of the THIN-B metallo-beta-lactamase of Janthinobacterium lividum. Antimicrob Agents Chemother. 2004; 48(12): 4778-83.

Fróes AM, da Mota FF, Cuadrat RRC, D'avila AMR. Distribution and classification of serine $\beta$-lactamases in Brazilian hospital sewage and other environmental metagenomes deposited in public databases. Front Microbiol. 2016; 7: 1-15.

Gibson MK, Forsberg KJ, Dantas G. Improved annotation of antibiotic resistance determinants reveals microbial resistomes cluster by ecology. ISME J. 2015; 9(1): 1-10.

Gudeta DD, Bortolaia V, Amos G, Wellington EM, Brandt KK, Poirel $\mathrm{L}$, et al. The soil microbiota harbors a diversity of carbapenemhydrolyzing $\beta$-lactamases of potential clinical relevance. Antimicrob Agents Chemother. 2016; 60(1): 151-60. 
Haack F, Poehlein A, Kröger C, Voigt C, Piepenbring M, Bode H, et al. Molecular keys to the Janthinobacterium and Duganella spp. Interaction with the plant pathogen Fusarium graminearum. Front Microbiol. 2016; 7: 1668.

Hornung C, Poehlein A, Haack F, Schmidt M, Dierking K, Pohlen A, et al. The Janthinobacterium sp. HH01 genome encodes a homologue of the $V$. cholerae CqsA and L. pneumophila LqsA autoinducer synthases. PLoS ONE. 2013; 8(2): e55045.

Kämpfer P, Rosselló-Mora R, Hermansson M, Persson F, Huber B, Falsen $\mathrm{E}$, et al. Undibacterium pigrum gen. nov., sp. nov., isolated from drinking water. Int J Syst Evol Microbiol. 2007; 57(Pt 7): 1510-5.

Katoh K, Rozewicki J, Yamada KD. MAFFT online service: multiple sequence alignment, interactive sequence choice and visualization. Brief Bioinform. 2017; doi: 10.1093/bib/bbx108.

Kim C, Villegas-Estrada A, Hesek D, Mobashery S. Mechanistic characterization of the bifunctional aminoglycoside-modifying enzyme AAC(3)-Ib/AAC(6')-Ib' from Pseudomonas aeruginosa. Biochemistry. 2007; 46(17): 5270-82.

Kumar S, Stecher G, Tamura K. MEGA7: Molecular Evolutionary Genetics Analysis Version 7.0 for Bigger Datasets. Mol Biol Evol. 2016; 33(7): 1870-4.

Liu W, Xie Y, Ma J, Luo X, Nie P, Zuo Z, et al. IBS: an illustrator for the presentation and visualization of biological sequences. Bioinformatics. 2015; 31(20): 3359-61.
Marchler-Bauer A, Bo Y, Han L, He J, Lanczycki CJ, Lu S, et al. CDD/ SPARCLE: functional classification of proteins via subfamily domain architectures. Nucleic Acids Res. 2017; 45(D1): D200-3.

Sigrist CJA, de Castro E, Cerutti L, Cuche BA, Hulo N, Bridge A, et al. New and continuing developments at PROSITE. Nucleic Acids Res. 2012; 41: D344-7.

Soucy SM, Huang J, Gogarten JP. Horizontal gene transfer: building the web of life. Nat Rev Genet. 2015; 16(8): 472-82.

Srivastava A, Singhal N, Goel M, Virdi JS, Kumar M. CBMAR: a comprehensive $\beta$-lactamase molecular annotation resource. Database (Oxford). 2014; 2014: bau111.

Wilke MS, Hills TL, Zhang HZ, Chambers HF, Strymadka NCJ. Crystal structures of the Apo and penicillin-acylated forms of the BlaR1 $\beta$-lactam sensor of Staphylococcus aureus. J Biol Chem. 2004; 279(45): 47278-87.

Yin C, Hulbert S, Schroeder K, Mavrodi O, Mavrodi D, Dhingra A, et al. Role of bacterial communities in the natural suppression of Rhizoctonia solani bare patch disease of wheat (Triticum aestivum L.). Appl Environ Microbiol. 2013; 79(23): 7428-38.

Zhang W, Fisher J, Mobashery S. The bifunctional enzymes of antibiotic resistance. Curr Opin Microbiol. 2009; 12(5): 505-11.

Zhou Y, Liang Y, Lynch K, Dennis JJ, David S, Wishart DS. PHAST: a fast phage search tool. Nucleic Acids Res. 2011; 39(2): W347-352. 